\title{
1 Endurance exercise ameliorates phenotypes in Drosophila models of
}

2 Spinocerebellar Ataxias

3

4

5

6

\section{4}

1- Department of Physiology, Wayne State University School of Medicine

2- Department of Pharmacology, Wayne State University School of Medicine

3- Department of Neurology, Wayne State University School of Medicine

*: Correspondence: 540 E Canfield, Scott Hall Rm 3108, Detroit, MI 48201, USA. stodi@wayne.edu, ${ }^{*} 540$ E Canfield, Scott Hall Rm 5275, Detroit, MI 48201, USA. rwessell@med.wayne.edu

Alyson Sujkowski ${ }^{1,2}$, Kristin Richardson ${ }^{1}$, Matthew V. Prifti ${ }^{2}$, R. J. Wessells ${ }^{1 * \star}$, Sokol V. Todi ${ }^{2,3^{\star}}$

$$
\text { rwessell@med.wayne.edu }
$$




\section{Abstract}

Endurance exercise is a potent intervention with widespread benefits proven to reduce disease incidence and impact across species. While endurance exercise supports neural

21 plasticity, enhanced memory, and reduced neurodegeneration, less is known about the effect of

22 chronic exercise on the progression of movement disorders such as ataxias. Here, we focused

23 on three different types of ataxias, Spinocerebellar Ataxias Type (SCAs) 2, 3, and 6, belonging

24 to the polyglutamine (polyQ) family of neurodegenerative disorders. In Drosophila models of

25 these SCAs, flies progressively lose motor function. Here, we observe marked protection of

26 speed and endurance in exercised SCA2 flies and modest protection in exercised SCA6

27 models, while no benefit is observed in SCA3 flies. Causative protein levels are reduced in

28 SCA2 flies after chronic exercise, but not in SCA3 models, linking protein levels to exercise-

29 based benefits. Additional investigations indicate that the exercise-inducible protein, Sestrin

30 (Sesn) suppresses mobility decline and improves early death in SCA2 flies, even without

31 exercise, coincident with disease protein level reduction and increased autophagic flux. These

32 improvements depend on previously established functions of Sesn that reduce oxidative

33 damage and modulate mTOR activity. Our study suggests differential responses of polyQ SCAs

34 to exercise, highlighting the potential for more extensive application of exercise-based therapies

35 in the prevention of polyQ neurodegeneration. Defining the mechanisms by which endurance

36 exercise suppresses polyQ SCAs will open the door for more effective treatment for these

37 diseases. 


\section{Introduction}

Nine inherited neurodegenerative disorders are caused by expansion of a CAG triplet

41 repeat in the protein-coding region of the respective disease genes. The CAG repeat encodes

42 the amino acid glutamine; thus, the disease protein carries a lengthened tract of polyglutamines

43 (polyQ) that renders it toxic. The polyQ family includes six Spinocerebellar Ataxias (SCAs 1, 2,

$443,6,7$, and 17), Huntington's Disease, Dentatorubral-pallidoluysian Atrophy, and Kennedy's

45 disease ${ }^{(1-7)}$. Various pathogenic mechanisms are shared, involving primarily toxic gain-of-

46 function acquired by the disease protein. These mutations yield aggregation of the toxic polyQ

47 protein and despite their wide expression throughout the body, only select central nervous

48 system regions degenerate in each disease. Other common mechanisms underlying polyQ

49 pathogenesis include mitochondrial, transcriptional, and autophagy dysfunction (8). Although

50 the disorders share disease mechanisms, the individual host proteins have divergent functions

$51(1-7)$

There is an unmet need for the treatment of polyQ diseases as there is currently no

53 therapeutic solution for any of these disorders. Here, we examined the idea that daily endurance

54 exercise might be of benefit against polyQ disorders. Endurance exercise is a low-cost, effective

55 intervention with broad pro-healthspan effects, including reducing the incidence of obesity, heart

56 disease, cancer, and cognitive defects (9-11). Previous studies examined to a limited extent the

57 effects of chronic exercise in patients with advanced stages of ataxia, including polyQ disorders:

58 some types of exercise might help in certain cases $(12-14)$ but not others $(15,16)$. These

59 studies may have been limited by lack of normalized protocols, genetics, and variable disease

60 stage among patients. Some work on the possible role of exercise was also conducted in

61 mouse models of HD, SBMA and SCA1. SCA1 and SBMA mice seemed to benefit from

62 exercise (17-20), whereas some models of HD improved $(21,22)$ while others did not (23). 
Differences in these studies could be due to variation in protocols, genetic

64 background, age of testing and other parameters difficult to normalize among studies.

65 While studies in animal models have identified potential pathways by which exercise

66 may affect polyQ phenotypes, absence of normalization may limit the overall applicability

67 and extension of those results to other polyQ diseases and further up the evolutionary

68 chain. Here, we take advantage of the Drosophila model system to systematically

69 examine which polyQ disorders are responsive to exercise therapy and to identify

70 mechanisms involved.

Drosophila melanogaster has been widely and successfully utilized to understand

72 polyQ disease mechanisms (24-27). In parallel, the fly has emerged as a highly efficient

73 model for studying long-term effects of chronic exercise (28). Following a 3-week daily,

74 gradually ramped training program, flies of diverse genotypes experience reproducible

75 changes to speed, endurance, flight, cardiac performance, mitophagy, and mitochondrial

76 function (29-36). These changes track with adaptive physiological changes seen in

77 chronically exercising humans $(28,37)$, and induce conserved changes in gene

78 expression, including conserved mediators of exercise benefits, such as PGC1-a and

79 Sesn $(38,39)$.

Here, we tested the effects of chronic exercise in three Drosophila models of

81 polyQ SCAs: 2, 3, and 6. Ectopic expression of these human, polyQ-expanded disease

82 proteins in flies is toxic (40-47). We find that exercise has a dramatically positive effect in

83 SCA2 flies, with modest effects on SCA6 model flies and no benefit to SCA3 flies,

84 indicating a range of response in different disease models. In addition, the exercise-

85 mimicking protein, Sesn improves early death and rescues mobility declines in SCA2

86 model flies. We further find that improvement in disease phenotypes depends on

87 functions of Sesn that reduce oxidative damage and modulate mTOR. Our observations 
88 identify Sesn as a key genetic mediator underlying the protective effects of exercise and

89 highlight mechanistic targets that can be leveraged towards therapeutic options for

90 patients unable to exercise, or to supplement the benefits of those who can.

\section{Results}

\section{Exercise differentially impacts mobility in Drosophila polyQ SCA models}

Expression of polyQ disease proteins in flies causes reduced motility and neuronal cell

94 death, as we and others have shown in various publications (24-27, 41, 45-52). To test if

95 endurance exercise reduces polyQ-dependent phenotypes in the fly, we selected three SCAs:

962,3 , and 6 (Figure 1A). Full-length, human disease protein was expressed through the binary,

97 RU486-inducible Gal4-UAS system $(32,53)$ in all fly neurons and only during adulthood, which

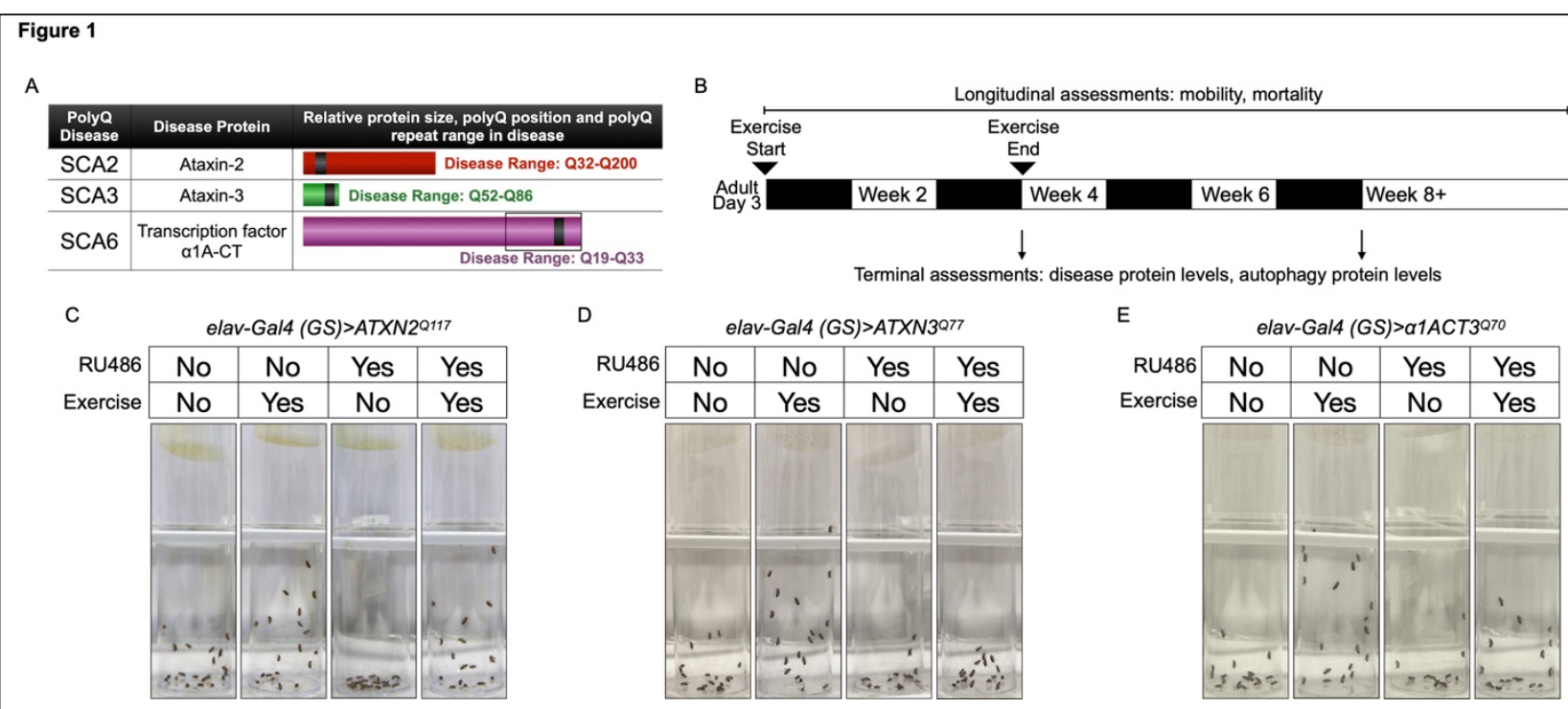

Figure 1: Endurance exercise differentially affects mobility in Drosophila models of Spinocerebellar Ataxia. (A) Table of Spinocerebellar Ataxia (SCA) models used in this study. (B) Timeline of endurance exercise program and assessment of physiology and disease protein levels. (C-E) Representative climbing speed images in Drosophila models of (C) SCA2, (D) SCA3 and (E) SCA6. Photos taken 2 seconds after inducing negative geotaxis response in 4-week-old flies, after endurance exercise is complete.

98 is toxic (40-47). We have established an optimized exercise protocol (Figure 1B) that drives

99 reproducible effects that are robust across genotypes $(29,34)$. These effects are not due to 
non-specific stress or behavioral effect of the regimen-unexercised flies exposed to training, but prevented from running, do not experience improvements $(29,34)$. unexercised siblings (28). SCA2 model flies expressing polyQ-expanded ATXN2 specifically in adult neurons (elav-Gal4 (GS)>ATXN2 ${ }^{\mathrm{Q117}}$ ) have reduced climbing speed by 4 weeks of age, but exercise fully rescues climbing speed to the level of age-matched, uninduced control flies that complete our three-week, ramped exercise program (Figure 1C, Supplementary video S1).

107 In contrast, four-week-old SCA3 model flies (elav-Gal4 (GS)>ATXN3 ${ }^{Q 77}$ ) have lower climbing 108 speed than unexercised, uninduced control flies and do not increase climbing speed after exercise training (Figure 1D). Flies expressing hyper-expanded $\alpha 1 \mathrm{ACT}$ (elav-Gal4

$110(G S)>\alpha 1 A C T Q^{70}$ ), the disease gene in SCA6 (44), also have reduced week-four climbing speed

111 in comparison to uninduced, unexercised control flies, but climbing speed is partially rescued 112 after exercise training (Figure 1E).

PolyQ disorders are progressive and neurodegenerative severity tends to increase with

114 age $(1,2)$. We have previously shown that exercise-induced improvements to climbing speed 115 and endurance are long-lasting and persist even after the three-week exercise program is 116 complete (29). Neither RU486 feeding nor expression of the empty vector used to generate

117 transgenic flies in adult neurons negatively affect exercise adaptations to either climbing speed 118 or endurance. Furthermore, expression of an essentially pure polyQ80 repeat (54) in adult 119 neurons reduced climbing speed and abrogated exercise benefits, while age-matched, 120 uninduced control flies adapted to exercise normally (Supplementary Figure S1A-C). Having confirmed that post-exercise differences in climbing speed can be discerned in

122 polyQ model flies, we next subjected the same three SCA models from Figure 1 to our 123 endurance exercise program and tested them longitudinally for changes to climbing speed and 124 endurance. In contrast to the ramped exercise program, endurance is tested by placing flies on 
125 the climbing apparatus on non-training days and allowing them to climb to exhaustion. When

126 fewer than $10 \%$ of flies in a vial no longer respond to the climbing stimulus, the time is recorded,

127 and the vial is removed from the apparatus and scored as fatigued. These data are plotted

128 similarly to a survival curve, with each datum representing a single vial of 20 flies (55).

SCA2 model flies have reduced climbing speed by adult week four (Figure 2A, compare

$130 \mathrm{RU}-\mathrm{UN}$ to RU+UN), but endurance exercise fully rescues climbing speed to the level of

131 exercised, uninduced control flies, and improvement persists into adult week five (Figure 2A,

132 compare RU-EX to RU+EX). Endurance in SCA2 model flies is similar to uninduced,

133 unexercised siblings (log-rank, $p=0.2018$ ) and exercised SCA2 model flies improve endurance

134 as well as exercised, uninduced controls (Figure 2B). We next examined ATXN2 protein levels

135 to see if improved climbing correlated with changes in toxic protein levels. Exercise markedly

136 reduced ATXN2 levels relative to age-matched, unexercised siblings, linking improved

137 physiology to reductions in disease protein (Figure 1C).

In contrast, SCA3 model flies do not improve climbing speed (Figure 2D) or endurance

139 (Figure 2E) after exercise training. Furthermore, exercised and unexercised SCA3 flies had

140 similar levels of ATXN3, the causative, polyQ-expanded disease protein in SCA3 (15) (Figure

$1412 \mathrm{~F})$. SCA6 model flies had reductions in climbing speed at weeks three and five compared to

142 unexercised, uninduced siblings, and exercise training improved climbing speed, albeit not to

143 the level of uninduced, exercised control flies (Figure 2G). Unexercised SCA6 flies trend toward

144 lower endurance relative to uninduced, unexercised control flies (log-rank, $p=0.0559$ ) and

145 improve endurance after exercise training, but do not equal the endurance of uninduced,

146 exercised controls (log-rank, $p=0.0218)$ (Figure 2H). 


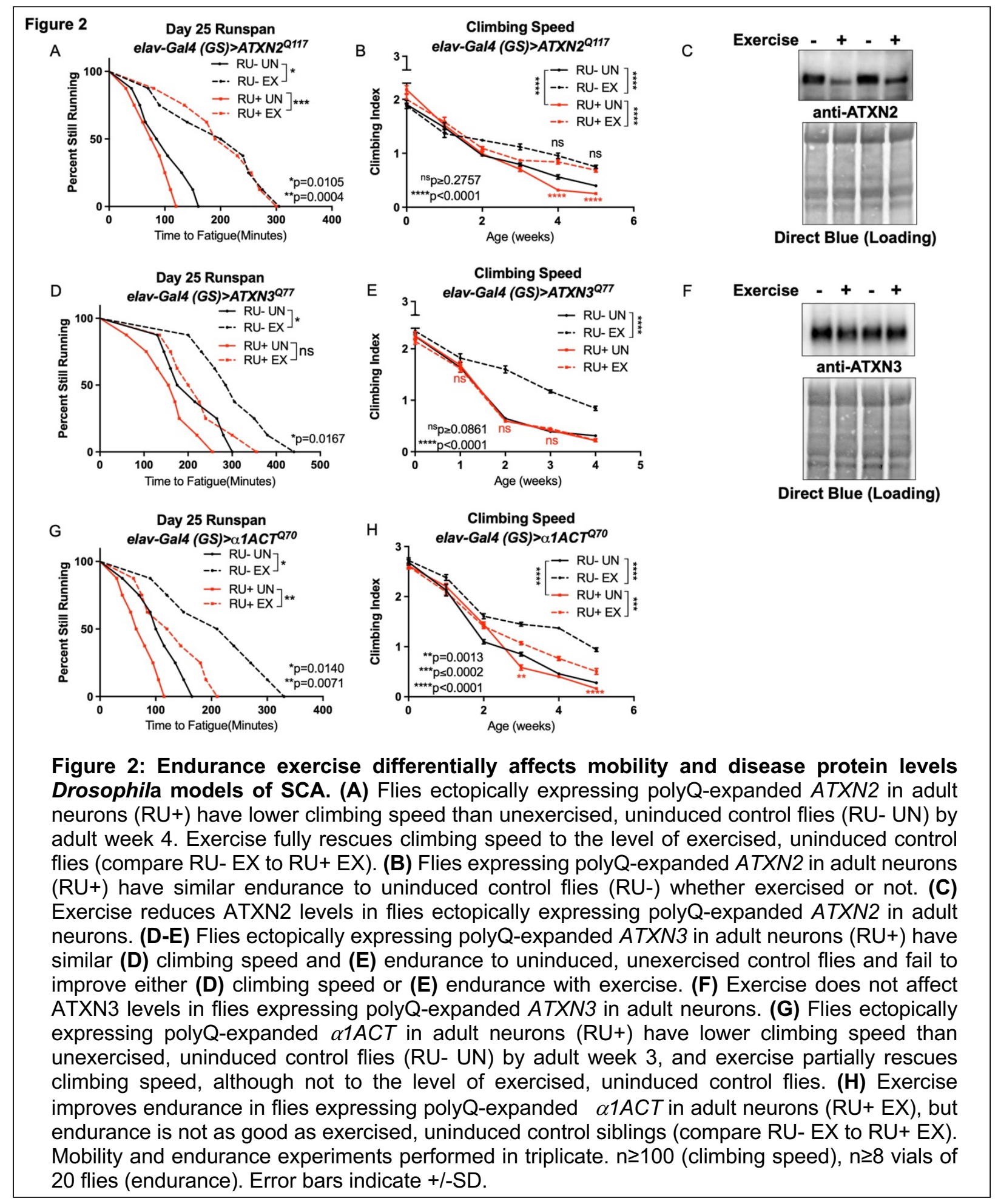

Sestrin expression improves phenotypes in SCA2 model flies without exercise. 

autophagy by inhibiting $\operatorname{mTOR}(32,57,58)$, and increased autophagy increases ATXN2
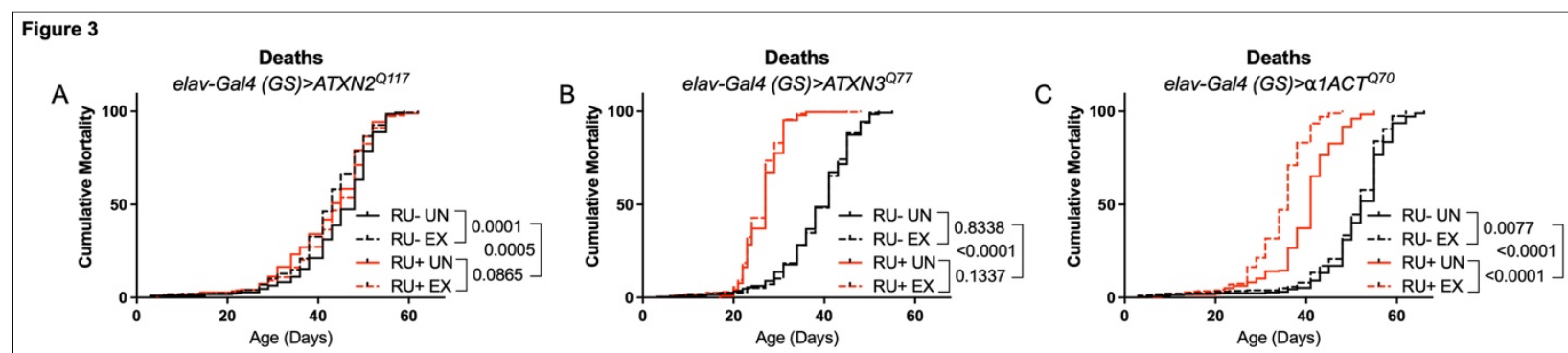

Figure 3: Expression of Atxn2 in adult neurons does not negatively impact lifespan. Cumulative mortality in (A) elav-Gal4 (GS) $>A T X N 2^{Q 117}$ flies is similar whether exercised or not. (B) elav-Gal4 (GS) $>A T X N 3^{Q 77}$ flies have reduced lifespan compared to uninduced controls, and exercise does not negatively affect either group. (C) elav-Gal4 (GS)>a1ACT ${ }^{\mathrm{T} 0}$ flies have lower lifespan than uninduced controls, and exercise reduces lifespan further. $n \geq 231$.

solubility and reduces phenotypes in SCA2 patient cells (59). Since muscle-specific dSesn

overexpression is sufficient to replicate the beneficial adaptations of endurance exercise, even in sedentary Drosophila (39), we next overexpressed dSesn in SCA2 model flies. selectively in adult neurons confirmed previously reported lifespan reductions in both SCA3 and SCA6 model flies $(44,60)$ (Figure 3B, C), but neither ATXN2 expression nor exercise significantly affected longevity in SCA2 flies (Figure 3A). ATXN2 CAG expansion causes early mortality in SCA2 patients (61); therefore, we performed subsequent experiments in flies ubiquitously expressing two copies of polyQ-expanded ATXN2 simultaneously with dSesn overexpression (sqh>ATXN2 ${ }^{Q 117} ; d S e s n^{W T} ; A T X N 2^{Q 117}$ ) to more closely model clinical subjects. Indeed, constitutive expression of polyQ-expanded ATXN2 in all tissues, throughout development and in adults, $\left(s q h>A T X N 2^{Q 117} ; A T X N 2^{Q 117}\right)$ caused early death in both female

167 (Figure 4A) and male (Figure 4D) flies. Mobility reductions appeared earlier than with panneuronal expression, present upon adult eclosion from the pupal case and persisting until death

169 (Figure 4B, E). On the other hand, flies ubiquitously expressing both ATXN2 ${ }^{\mathrm{Q} 117}$ and dSesn 170 improved survival (Figure 3A, D), although rescue was not to the level of background control

171 flies. Mobility improvements were more pronounced, with complete rescue of climbing speed in 
172 both females (Figure 3B) and males (Figure 3E). We next examined disease protein levels in

173 sqh>ATXN2 $2^{Q 117} ;$ dSesn ${ }^{W T} ; A T X N 2^{Q 117}$ flies and found significant reduction in ATXN2 compared to

174 SCA2 flies without dSesn expression (Figure 4C, F, quantified in Figure 7), similar to

175 aforementioned reduction in disease protein after exercise. In contrast, ubiquitous expression of

176 either $\mathrm{ATXN3}{ }^{\mathrm{Q77}}$ or $\alpha 1 \mathrm{ACT} \mathrm{T}^{\mathrm{Q70}}$ leads to developmental and early adult lethality $(44,60)$, and

177 dSesn expression did not improve outcomes (data not shown).

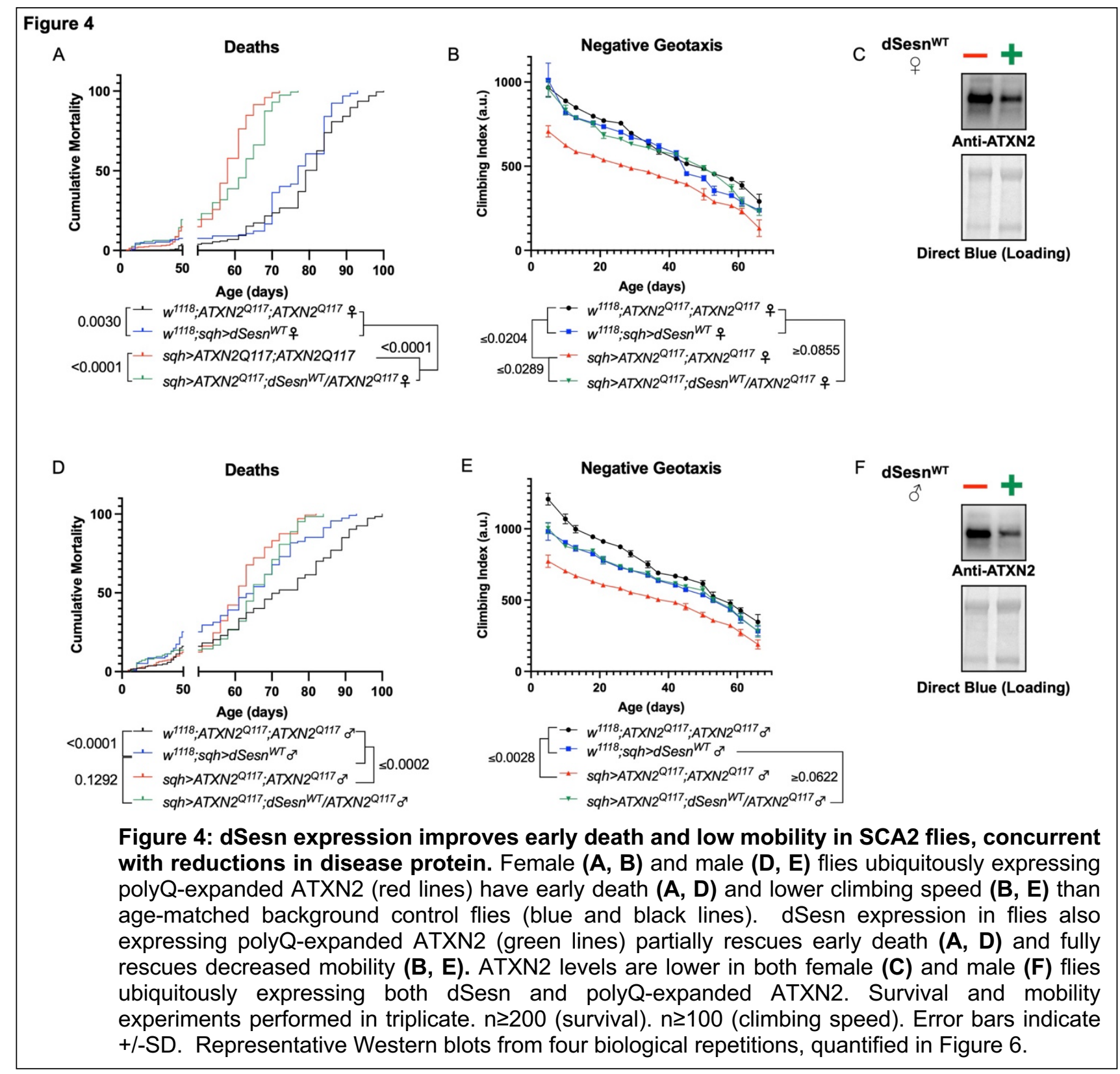



activity while the D424A (dSesn ${ }^{\mathrm{DA}}$ ) and D423A/D424A (dSesn ${ }^{\mathrm{DDAA}}$ ) substitutions disrupt TORC1 interaction with mTOR is required for the mobility-extending effects of dSesn in wild-type,

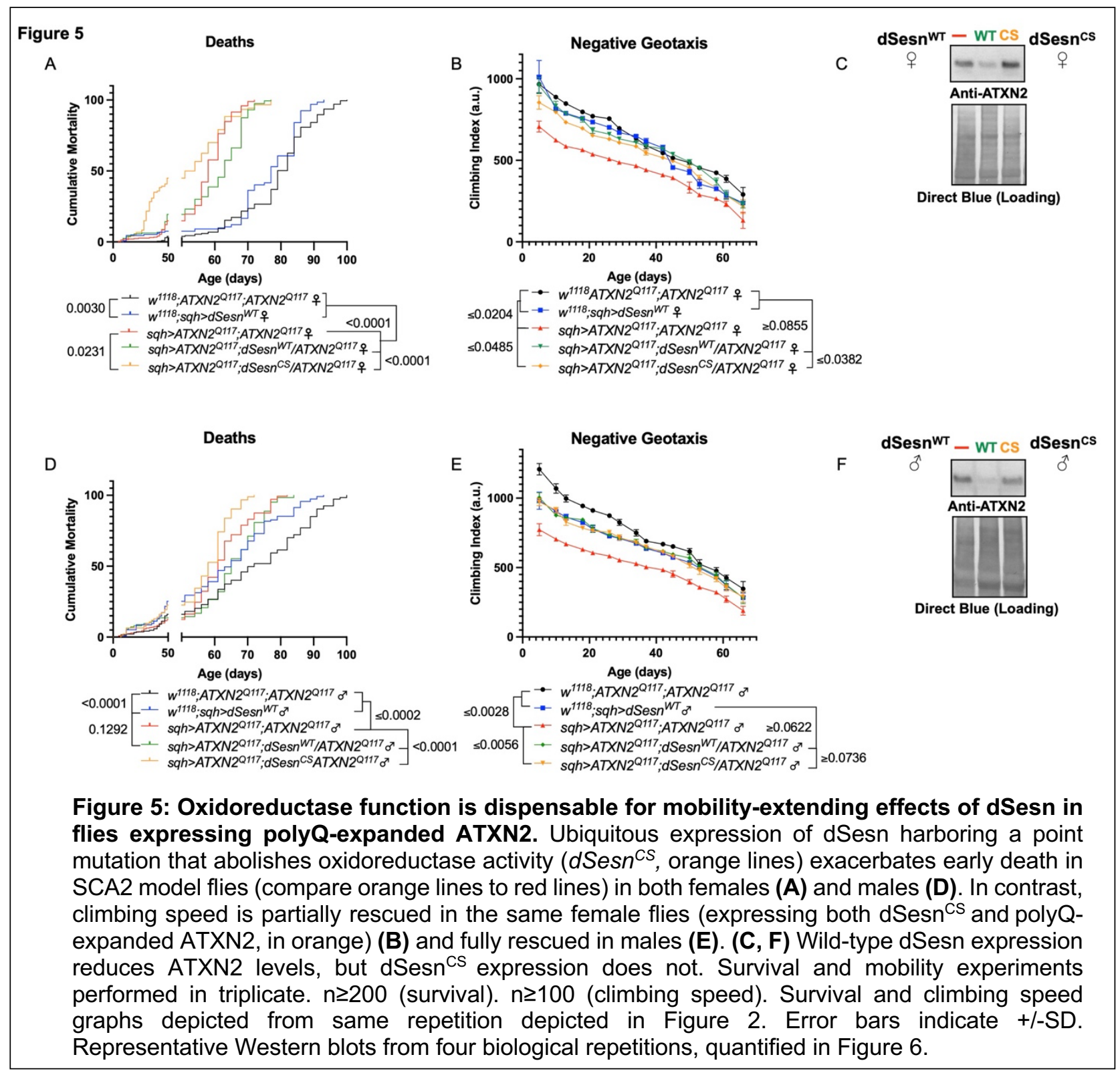


sedentary flies (39). Overexpression of either wild-type or mutated dSesns does not increase

187 longevity in a wild-type background (i.e. without polyQ protein expression; Supplementary

188 Figure S2). To explore the requirement of Sesn's various functions for suppression of polyQ

189 phenotypes, we performed mobility, survival and protein expression experiments in which we

190 ubiquitously overexpressed dSesn ${ }^{\mathrm{CS}}, \mathrm{dSesn}{ }^{\mathrm{DA}}, \mathrm{dSesn^{ \textrm {DDAA } }}{ }^{\text {, or wild-type dSesn (dSesn }}{ }^{\mathrm{WT}}$ ) in

191 SCA2 flies.

First, we overexpressed dSesn without its antioxidant function $\left(s q h>A T X N 2^{Q 117} ; d S e s n^{C S} ; A T X N 2^{Q 117}\right)$. Ubiquitous expression of dSesn ${ }^{c S}$ in SCA2 model flies

194 does not provide any protection - in fact, it exacerbates premature death in both male and

195 female flies (Figure 5A, D). However, we observed pronounced mobility improvements in female

196 flies (Figure 5B) and mobility is fully rescued in males (Figure 5E). Whereas wild-type dSesn

197 overexpression reduces ATXN2 levels in both female (Figure 5C) and male (Figure 5F) SCA2

198 model flies, dSesn ${ }^{\mathrm{CS}}$ overexpression does not have the same impact (Figure 5C, F, quantified in

199 Figure 7). These results suggest that while the oxidoreductase function of dSesn is not required

200 for protection against motility defects in SCA2 flies, it is essential for improved survival.

201 We next ubiquitously expressed two separate amino-acid substitutions of dSesn that

202 knock out its ability to modulate mTOR (62). Like dSesn ${ }^{c S}$ experiments, expression of either

203 dSesn ${ }^{\text {DA }}$ (Figure 6A-D) or dSesn ${ }^{\text {DDAA }}$ (Figure 6E-H) increases early death in both female (Figure

$2046 \mathrm{~A}, \mathrm{E}$ ) and male (Figure 6C, G) SCA2 model flies. In female flies, both dSesn ${ }^{\mathrm{DA}}$ and dSesn ${ }^{\text {DDAA }}$

205 confer modest improvements to climbing speed compared to SCA2 model flies, but

206 improvement does not equal that of wild type dSesn expression and is absent at later timepoints

207 (Figure 6B, F). Male flies ubiquitously expressing dSesn ${ }^{\mathrm{DA}}$ does not rescue mobility defects at

208 any age (Figure 6D), while overexpression 


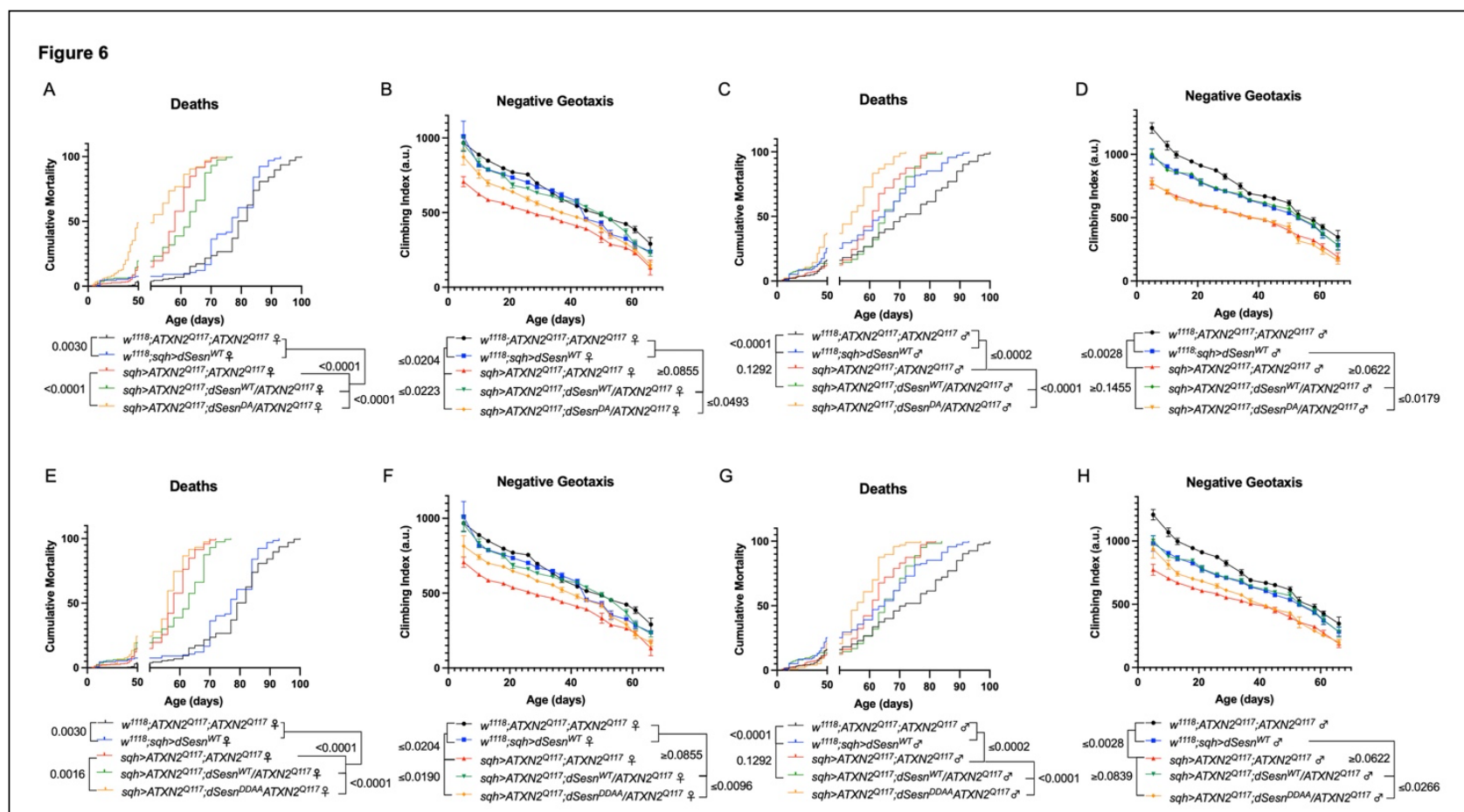

Figure 6: Interaction with mTOR is required for dSesn to improve survival and mobility in flies expressing polyQ-expanded ATXN2. Ubiquitous expression of dSesn harboring two separate mutations that abolish mTORC interaction $d \operatorname{Sesn}^{D A}(\mathbf{A}-\mathrm{D}), d \operatorname{Sesn}^{D D A A}(\mathrm{E}-\mathrm{H})$ orange lines) exacerbates early death (A,E, females, $\mathbf{C}, \mathbf{G}$, males) and fails to rescue mobility $(\mathbf{B}, \mathbf{F}$, females, D,H, males) in SCA2 model flies. Survival and mobility experiments performed in triplicate. Survival and climbing speed graphs depicted from same repetition depicted in Figure 2. $n \geq 200$ (survival). $\mathrm{n} \geq 100$ (climbing speed). Error bars indicate +/-SD. of $d$ Sesn $^{\text {DDAA }}$ provides modest climbing speed improvements at early ages only (Figure $6 \mathrm{H}$ ).

211 Thus, interaction with mTOR appears critical for full rescue of climbing speed and protection against early death in SCA2 model flies.

To examine the relative impact of dSesn expression on disease protein levels, we again

214 performed Western blots on flies ubiquitously expressing polyQ-expanded ATXN2 with and

215 without simultaneous expression of either wild type dSesn or dSesn harboring mutations that

216 separately abolish its oxidative protection or mTOR modulating functions. ATXN2 protein in

217 SCA2 model flies (sqh>ATXN2 ${ }^{Q 117} ; A T X N 2^{Q 117}$ ) was easily visible by log-phase of mortality

218 (Figure 7A-C, F). Although all dSesn-expressing cohorts had variable reductions in ATXN2

219 levels, only expression of wild-type dSesn correlated with significantly reduced disease protein.

220 These data may reflect different mechanisms of protection at play and are discussed further

221 below. 

muscle-specific dSesn overexpression $(39,63)$, we hypothesized that dSesn overexpression and reduced ATXN2 levels relate to changes in autophagy. We observed that Atgla/lla ratios increased in SCA2 model flies that were overexpressing wild-type dSesn, but not its mutated versions, which trend toward increased autophagy without reaching significance (Figure 7C, D-

$227 \mathrm{~F})$.

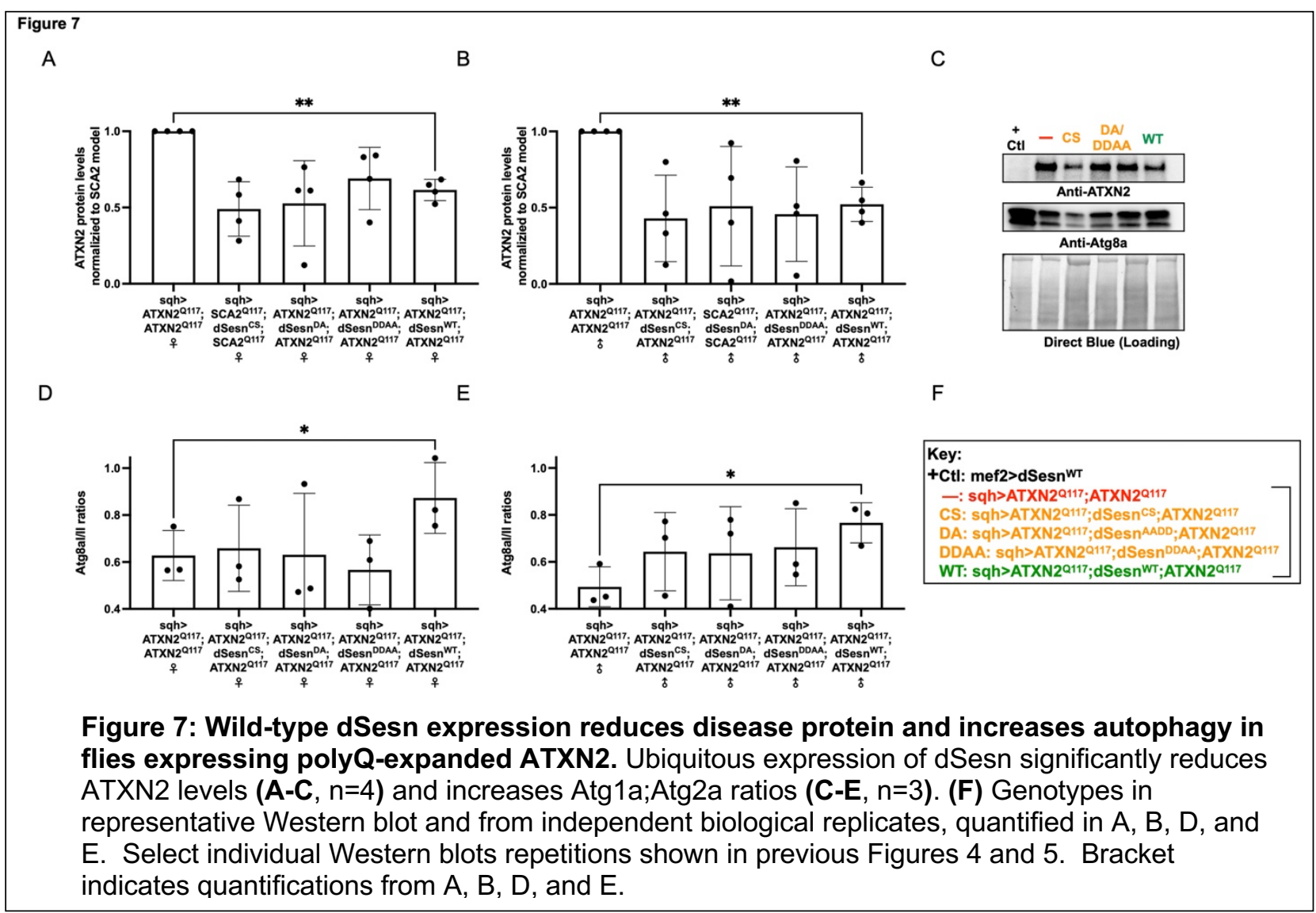

Finally, since dSesn overexpression improved survival in flies ubiquitously expressing

229 two copies of the polyQ-expanded ATXN2 transgene, we repeated our longevity experiments in

230 this model of exercised SCA2 flies. Exercised female flies expressing two copies of polyQ-

231 expanded ATXN2 either ubiquitously (Figure 8A) or in adult neurons (Figure 8C) significantly

232 improved survival over unexercised siblings. Male SCA2 model flies trended toward improved

233 survival in early life only, while they were still being subjected to exercise training (Figure 8B, D). 
234 Altogether, these findings indicate protective effects from endurance exercise not only in terms

235 of motility (Figures 1, 3) but also in terms of longevity (Figure 8) in SCA2 flies.

In summary, both exercise and dSesn expression ameliorate mobility and survival

237 defects in SCA2 model flies and these improvements correlate with reduced disease protein

238 levels. Furthermore, these neuroprotective effects require known functions of dSesn related to

239 mTOR and oxidoreductase activities.

\section{Discussion}

242 conditions and diseases, but, until now, has not been systematically examined across several

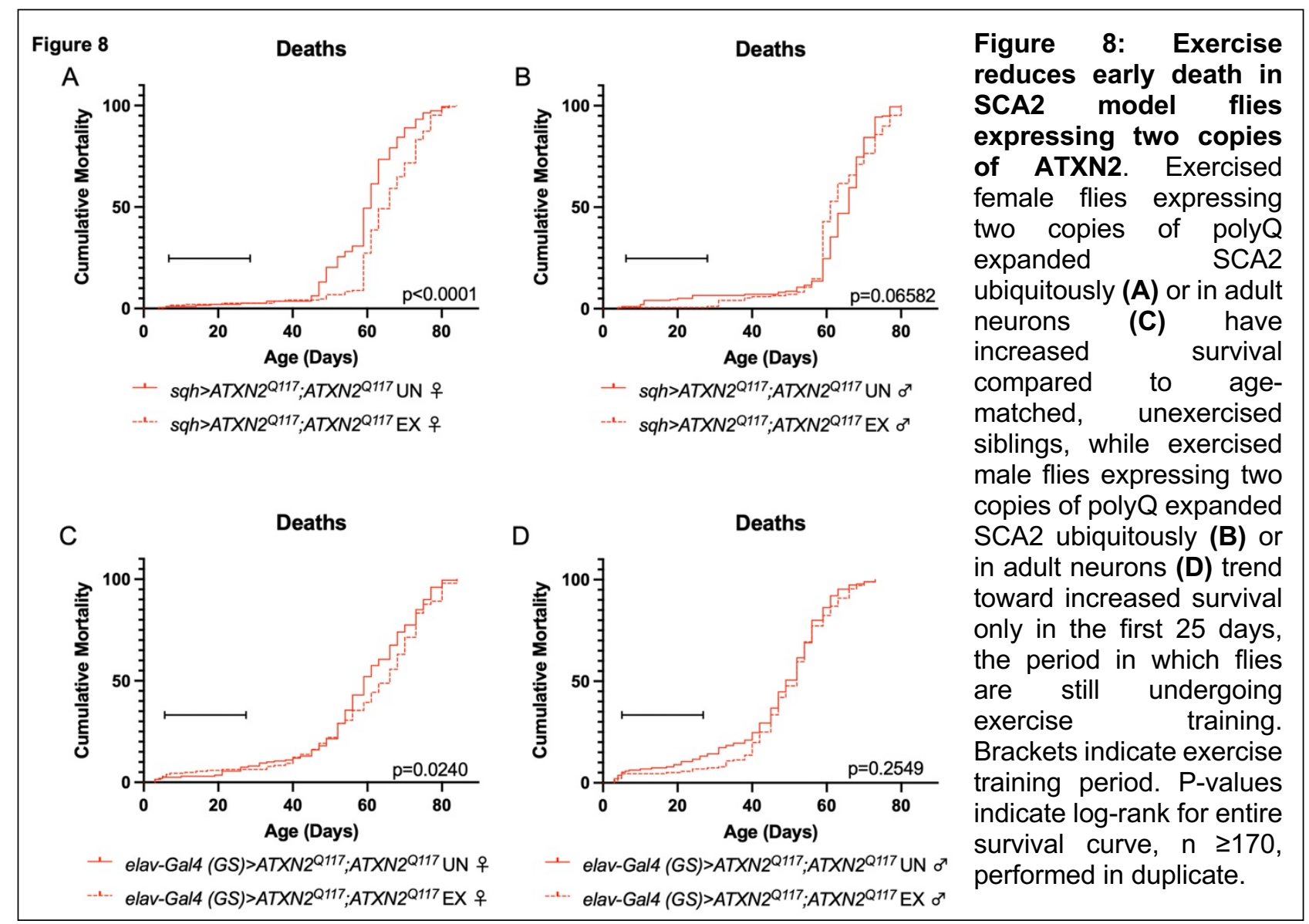

243 polyQ disorders. Lack of systematic studies in this respect also means that exercise-dependent

244 molecular mechanisms that can be utilized to fight polyQ diseases remain largely unknown. 
245 Here, we explored in a highly controlled and rigorously quantified way the possibility that daily

246 training is of benefit against some polyQ diseases, but not necessarily others. In addition, we

247 identified key mediators of exercise's protective effects that can be harvested towards

248 therapeutic options for individuals unable to exercise, or to enhance benefits in those who can.

249 Endurance exercise significantly rescued progressive motility defects in SCA2 flies,

250 allowing their climbing speed and endurance to remain in wild-type range. Exercise also partially

251 rescued SCA6 flies, with no beneficial effect on SCA3 flies. Thus, exercise can have potent

252 rescue effects for particular polyQ diseases. To obtain early insight into how exercise could lead

253 to such dramatic improvement in SCA2 flies (albeit not for SCA3) we examined protein levels of

254 ataxin-2 and ataxin-3, their respective disease proteins. Exercise led to clear reduction in

255 protein levels of ataxin-2, but did not noticeably affect ataxin-3. In the context of endurance

256 exercise, improved motility in SCA2 flies may be due to reduced disease protein levels.

In addition, we examined the effect of the powerful exercise-mimetic protein Sesn on

258 lifespan, motility and disease protein levels in SCA2 model flies. Sesns are induced by oxidative

259 and genotoxic stress in flies and mammals and promote autophagy $(58,64)$. Sesns protect

260 against oxidative stress-induced fly neuronal death (65) and reduce accumulation of aggregates

261 in mammalian cells (66). In previous work with wild-type flies, we found that expression of fly

262 Sesn is sufficient to preserve speed and endurance and has no additive effect with exercise,

263 consistent with a role as a key effector of exercise (39). Here, we found that Sesn was

264 protective in SCA2 model flies, but did not confer benefit to either SCA3 or SCA6. Furthermore,

265 while Sesn's oxidoreductase function was not necessary to rescue mobility defects in SCA2,

266 both oxidative resistance and mTOR modulating activities were required for Sesn to improve

267 early death. In fact, overexpression of Sesn with mutations eliminating either its oxidoreductase

268 activity or mTOR modulating functions exacerbated premature death in SCA2 flies. 


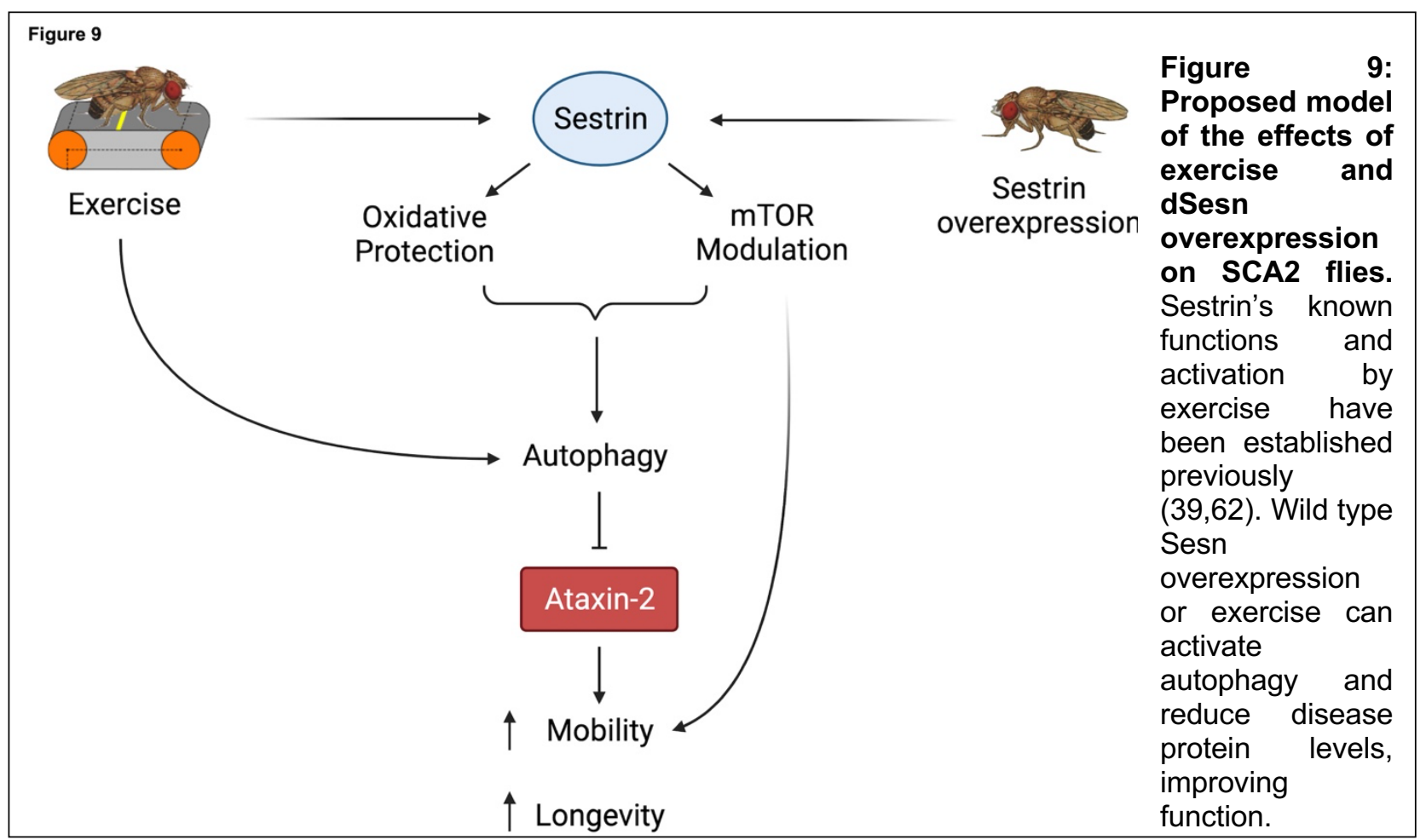

269 We have previously shown that the oxidoreductase activity of Sesn is dispensable for

270 motility improvements in wild-type flies (39), similar to our observations with SCA2 flies in this

271 study. Sesn overexpression is also not sufficient to extend lifespan in a wild-type background,

272 shown here and elsewhere $(39,58)$. Neurons are particularly susceptible to oxidative stress and

273 reactive oxygen species can induce cell death during neurodegeneration (67). Perhaps, in the

274 context of neurodegenerative disease, Sesn protects mobility until oxidative burden in neurons

275 becomes insurmountable.

Wild-type Sesn overexpression in SCA2 model flies correlates with significantly reduced

277 disease protein and increased autophagic flux. Overexpression of mutated versions of Sesn led

278 to reduced disease protein levels without reaching statistical significance. This is not entirely

279 surprising: mutated Sesns still had some positive impact on SCA2 fly mobility, but did not have

280 the same, powerful protective effect of wild-type Sesn. Additionally, mutant Sesns, as

281 mentioned above, failed to improve lethality in SCA2 model flies. Collectively, these findings

282 suggest separate mechanisms at play: some that may depend on reduced toxic protein levels 
283 (as observed before in a model of HD and exercise $(68,69)$ ), and others that may protect

284 independently of changes in degenerative protein levels. Future investigations are required to

285 parse out such mechanisms and whether they function in a cell autonomous or non-cell

286 autonomous manner.

To conclude, we propose endurance exercise as a promising therapeutic intervention for polyQ neurodegeneration, particularly in SCA2. We also identify Sesn as a genetic mediator of exercise-induced improvements in SCA2 phenotypes and demonstrate that Sesn can substitute

290 for exercise benefits, even in flies that do not exercise. Identification of molecular targets

291 capable of inducing exercise-like enhancements in the presence of neurodegeneration is

292 particularly important, as many patients will eventually lose the ability to exercise. This work has

293 begun to uncover exercise-induced mechanisms that can be utilized for neuroprotection against

294 specific polyQ diseases and can be leveraged toward therapeutics in the future.

\section{Methods}

\section{Antibodies}

Primary antibodies were obtained from the following sources: anti-ataxin-2 (mouse monoclonal, 1:500, BD biosciences) anti-ataxin-3 (mouse monoclonal 1H9, MAB5360, 1:500_

299 1000; Millipore), anti-MJD (rabbit polyclonal, 1:15,000)(70)), anti-dSesn (rabbit polyclonal,

300 1:500, (58)), anti-GABARAP (rabbit polyclonal, Abcam, 1:1000) Peroxidase conjugated

301 secondary antibodies (goat anti-mouse, goat anti-rabbit, 1:5000; Jackson Immunoresearch). 
Drosophila Stock Center (Bloomington, IN). Both SCA2 lines were used for pilot work. A single line containing UAS-ATXN2 ${ }^{\mathrm{Q} 117}$ on both chromosomes II and III was then generated using standard crosses (w-;UAS-ATXN2 ${ }^{\mathrm{Q} 117} /$ CyO;UAS-ATXN2 ${ }^{\mathrm{Q} 117}$ ).

Wild-type (dSesn $\left.{ }^{\text {WT }}\right)$ and C86S(dSesn $\left.{ }^{\mathrm{CS}}\right)$, D424A (dSesn $\left.{ }^{\mathrm{DA}}\right)$ or D423A/D424A

$311\left(\mathrm{dSesn}{ }^{\mathrm{DDAA}}\right)$ mutations are described in $(39,62)$. C86, D423 and D424 in dSesn correspond to

312 C125, D406 and D407 in mammalian SESN2 (62). These four lines were separately

313 recombined with sqh-Gal4 to create four independent lines with genotypes: +;sqh/CyO;UAS-

$314 \mathrm{dSesn} \mathrm{XX}^{\mathrm{X}} \mathrm{TM} 3-\mathrm{Sb}$. Simultaneous overexpression of both UAS-ATXN2 ${ }^{\mathrm{Q} 117}$ and UAS-dSesn ${ }^{\mathrm{XX}}$ was

315 achieved via a single cross between w-; UAS-ATXN2 ${ }^{\mathrm{Q} 117} / \mathrm{CyO}$;UAS-ATXN2 ${ }^{\mathrm{Q} 117}$ virgin females

316 and +;sqh/CyO;UAS-dSesn ${ }^{\mathrm{XX}} / \mathrm{TM} 3-\mathrm{Sb}$ males to generate +;UAS-ATXN2 ${ }^{\mathrm{Q} 117}$;UAS-

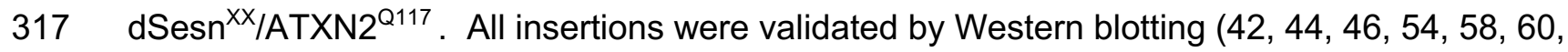
$31871,72)$

Prior to all experiments, fly cultures were maintained at a constant density for at least two generations. $20-25$ virgin females (depending on genotype) and 5 males were mated in 300

$321 \mathrm{~mL}$ bottles with $50 \mathrm{~mL}$ standard $10 \%$ sucrose $10 \%$ yeast spiked with $500 \mu \mathrm{L}$ Penicillin-

322 Streptomycin $(10,000 \mathrm{u} / \mathrm{mL}, 10 \mathrm{mg} / \mathrm{mL}$ in $0.9 \%$ sterile $\mathrm{NaCl}$ (Sigma Aldrich). Adult progeny were 323 synchronized by collecting within 6 hours of eclosion over a 24 hour time period. Groups of 20

324 age and sex-matched flies were immediately transferred into narrow polypropylene vials

325 containing $5 \mathrm{~mL}$ of standard $10 \%$ sucrose $10 \%$ yeast (no antibiotic) or RU486 food as

326 appropriate. Food vials were changed every day during exercise and every second day

327 thereafter and scored for mobility and lifespan until no flies remain.

Flies were housed in a $25^{\circ} \mathrm{C}$ incubator on a $12: 12$ light:dark cycle at $50 \%$ relative

329 humidity. Control flies for all non-gene-switch Gal4 UAS experiments consisted of both the UAS

330 and Gal4 lines into $w^{1118}$. For gene-switch experiments, RU- flies of the same genotype served

331 as the negative control. RU+ group received $100 \mu \mathrm{M}$ RU486/mifepristone (Cayman Chemical, 
332 Ann Arbor, MI), which activates the gene switch (GS) driver, while RU- group received the same

333 volume of vehicle solution $(70 \%$ ethanol).

334 Exercise Training

Triplicate cohorts of at least 800 flies were collected under light $\mathrm{CO}_{2}$ anesthesia within 6

336 hours of eclosion and separated into vials of 20. Flies were then further separated into 2 large

337 cohorts of at least 400 flies, which served as exercised and unexercised groups. Exercised

338 groups received three weeks of ramped exercise as described (29). The unexercised groups

339 were placed on the exercise training device at the same time as the exercised groups but were

340 prevented from running by the placement of a foam stopper low in the vial. Both cohorts were

341 housed in the same incubator with normal foam stopper placement at all times other than during

342 an exercise bout.

343 Climbing Speed

$344 \quad$ Negative geotaxis was assessed in Rapid Negative Geotaxis (RING) assays in groups of

345 at least 100 flies as described (73). Briefly, vials of 20 flies were briskly tapped down, then

346 measured for climbing distance after 2 s of inducing the negative geotaxis instinct. For each

347 group of vials, an average of 5 consecutive trials was calculated and batch processed using

348 ImageJ (Bethesda, MD). Flies were longitudinally tested 2-3 times per week for 5-7 weeks.

349 Between assessments, flies were returned to food vials and housed normally as described

350 above. Negative geotaxis results were analyzed using two-way ANOVA analysis (age-effect,

351 genotype effect) with post hoc Tukey multiple comparison tests in GraphPad Prism (San Diego,

352 CA, USA). All negative geotaxis experiments were performed in triplicate, with one complete

353 trial shown in each graph.

354 Endurance 
At least eight vials of 20 flies from each cohort were subjected to the endurance analysis on day 25 of adulthood, immediately after exercise was complete and after 2 days of recovery.

357 For each session, the flies were placed on the Power Tower exercise machine (28) and the

358 climbing instinct was induced until flies no longer responded to the negative geotaxis stimulus.

359 Monitored at 15 min intervals, a vial of flies was visually determined to be fatigued when $10 \%$ or

360 fewer flies could climb higher than $1 \mathrm{~cm}$ after three consecutive drops. Each vial was plotted as

361 a single datum. Endurance experiments were performed in triplicate and at the same time as

362 age-matched background controls and were scored blindly when possible. The time from the

363 start of the assay to the time of fatigue was recorded for each vial, and the data analyzed using

364 log-rank analysis in GraphPad Prism (San Diego, CA, USA). Each graph represents an

365 independent, representative repetition.

366 Longevity

For exercise experiments, appropriate food vials (RU486, vehicle or standard $10 \%$ sucrose $10 \%$ yeast) were changed and deaths were recorded five times per week during exercise training and three times per week after completion of the training program. All other

370 longevity experiments were scored three times per week. Dead flies were removed and

371 counted until no flies remained. Differences in survival were plotted as probability of death and

372 assessed following censoring using log-rank analysis in GraphPad Prism. Longevity

373 experiments were performed in triplicate and in parallel with background controls, with each

374 individual graph depicting a representative biological repetition.

Three to five whole flies per biological replicate, depending on experiment, were

377 homogenized in boiling lysis buffer (50 mM Tris pH 6.8, 2\% SDS, 10\% glycerol, $100 \mathrm{mM}$

378 dithiothreitol), sonicated, boiled for $10 \mathrm{~min}$, and centrifuged at 13,300xg at room temperature for $37910 \mathrm{~min}$. Western blots were developed ChemiDoc (Bio-Rad) and quantified with ImageLab (Bio- 
Rad). For direct blue staining, PVDF membranes were submerged for 10 min in $0.008 \%$ Direct Blue 71 (Sigma-Aldrich) in 40\% ethanol and 10\% acetic acid, rinsed in $40 \%$ ethanol $/ 10 \%$ acetic acid, air dried, and imaged. Western blots were performed using at least 3 biological replicates, and statistical analysis was performed in GraphPad Prism.

\section{Statistical Analysis}

Survival and endurance analyses were analyzed by log-rank. Non-natural deaths were censored and did not exceed $10 \%$ of flies for any group. Climbing speed over time was analyzed by Two-way ANOVA for age effect and genotype effect, with Tukey post-hoc comparison between significantly different groups. Western blots were analyzed using either

389 student t-test or ANOVA depending on experiment. All statistics were performed using

390 GraphPad Prism software version 9.2.0 for Macintosh.

\section{$391 \quad$ Acknowledgements}

392 We thank Henry Paulson for the anti-MJD antibody, and Todi lab members Kozeta Libohova,

393 Wei-Ling Tso, Gorica Ristic, Joanna Sutton, Jessica Blount, and Sean Johnson for generation 394 and validation of the SCA lines used in this study. We acknowledge our collaborators Myungjin 395 Kim and Jun Hee Lee for the original dSesn Drosophila stocks and reagents.

\section{Conflict of Interest Statement}

397 The authors declare no conflict of interest. The funders had no role in the design of the study; in 398 the collection, analyses, or interpretation of data; in the writing of the manuscript, or in the 399 decision to publish the results. 
bioRxiv preprint doi: https://doi.org/10.1101/2021.12.06.471345. this version posted December 7. 2021. The copyright holder for this preprint (which was not certified by peer review) is the author/funder, who has granted bioRxiv a license to display the preprint in perpetuity. It is made available under aCC-BY 4.0 International license.

402 This research was funded by the Wayne State University Thomas C. Rumble Graduate

403 Fellowship (to ALS), NIH R01 AG059683 (to RJW), NIH R21 NS121276 (to RJW and SVT)

404 and NIH R01 NS086778 (to SVT).

405

406

407

408

409 
4121 Zoghbi, H.Y. and Orr, H.T. (2000) Glutamine repeats and neurodegeneration. Annu Rev

413 Neurosci, 23, 217-247.

4142 La Spada, A.R. and Taylor, J.P. (2003) Polyglutamines placed into context. Neuron, 38, $415 \quad 681-684$.

4163 Todi, S.V., Williams, A. and Paulson, H. (2007) Waxman, S.G. (ed.), In Molecular

417 Neurology. Academic Press, London, in press., pp. 257-276.

4184 Zoghbi, H.Y. and Orr, H.T. (2009) Pathogenic mechanisms of a polyglutamine-mediated

419 neurodegenerative disease, spinocerebellar ataxia type 1. J Biol Chem, 284, 7425-7429.

4205 Paulson, H.L., Shakkottai, V.G., Clark, H.B. and Orr, H.T. (2017) Polyglutamine

421 spinocerebellar ataxias - from genes to potential treatments. Nat Rev Neurosci, 18, 613-626.

4226 Lieberman, A.P., Shakkottai, V.G. and Albin, R.L. (2018) Polyglutamine Repeats in

423 Neurodegenerative Diseases. Annu Rev Pathol, in press.

4247 Buijsen, R.A.M., Toonen, L.J.A., Gardiner, S.L. and van Roon-Mom, W.M.C. (2019)

425 Genetics, Mechanisms, and Therapeutic Progress in Polyglutamine Spinocerebellar Ataxias.

426 Neurotherapeutics, in press.

4278 Sullivan, R., Yau, W.Y., O'Connor, E. and Houlden, H. (2019) Spinocerebellar ataxia: an 428 update. J Neurol, 266, 533-544.

4299 Booth, F.W., Roberts, C.K. and Laye, M.J. (2012) Lack of exercise is a major cause of 430 chronic diseases. Compr Physiol, 2, 1143-1211.

43110 Wilmot, E.G., Edwardson, C.L., Achana, F.A., Davies, M.J., Gorely, T., Gray, L.J.,

432 Khunti, K., Yates, T. and Biddle, S.J. (2012) Sedentary time in adults and the association with 433 diabetes, cardiovascular disease and death: systematic review and meta-analysis. Diabetologia, $434 \quad 55,2895-2905$. 
43511 Strasser, B. (2013) Physical activity in obesity and metabolic syndrome. Ann N Y Acad

436 Sci, 1281, 141-159.

43712 Chang, Y.J., Chou, C.C., Huang, W.T., Lu, C.S., Wong, A.M. and Hsu, M.J. (2015)

438 Cycling regimen induces spinal circuitry plasticity and improves leg muscle coordination in

439 individuals with spinocerebellar ataxia. Arch Phys Med Rehabil, 96, 1006-1013.

44013 Schatton, C., Synofzik, M., Fleszar, Z., Giese, M.A., Schols, L. and Ilg, W. (2017)

441 Individualized exergame training improves postural control in advanced degenerative

442 spinocerebellar ataxia: A rater-blinded, intra-individually controlled trial. Parkinsonism Relat

443 Disord, 39, 80-84.

44414 de Oliveira, L.A.S., Martins, C.P., Horsczaruk, C.H.R., da Silva, D.C.L., Vasconcellos,

445 L.F., Lopes, A.J., Meira Mainenti, M.R. and Rodrigues, E.C. (2018) Partial Body Weight-

446 Supported Treadmill Training in Spinocerebellar Ataxia. Rehabil Res Pract, 2018, 7172686.

44715 D'Abreu, A., Franca, M.C., Jr., Paulson, H.L. and Lopes-Cendes, I. (2010) Caring for

448 Machado-Joseph disease: current understanding and how to help patients. Parkinsonism Relat

449 Disord, 16, 2-7.

45016 Wang, R.Y., Huang, F.Y., Soong, B.W., Huang, S.F. and Yang, Y.R. (2018) A

451 randomized controlled pilot trial of game-based training in individuals with spinocerebellar ataxia

452 type 3. Sci Rep, 8, 7816.

45317 Fryer, J.D., Yu, P., Kang, H., Mandel-Brehm, C., Carter, A.N., Crespo-Barreto, J., Gao,

454 Y., Flora, A., Shaw, C., Orr, H.T. et al. (2011) Exercise and genetic rescue of SCA1 via the

455 transcriptional repressor Capicua. Science, 334, 690-693.

45618 Chuang, C.S., Chang, J.C., Soong, B.W., Chuang, S.F., Lin, T.T., Cheng, W.L., Orr, H.T.

457 and Liu, C.S. (2019) Treadmill training increases the motor activity and neuron survival of the

458 cerebellum in a mouse model of spinocerebellar ataxia type 1. Kaohsiung J Med Sci, 35, 679-

459685. 

and Lieberman, A.P. (2014) Transcriptional activation of TFEB/ZKSCAN3 target genes

462 underlies enhanced autophagy in spinobulbar muscular atrophy. Hum Mol Genet, 23, 13764631386.

46420 Giorgetti, E., Yu, Z., Chua, J.P., Shimamura, R., Zhao, L., Zhu, F., Venneti, S., Pennuto,

465 M., Guan, Y., Hung, G. et al. (2016) Rescue of Metabolic Alterations in AR113Q Skeletal

466 Muscle by Peripheral Androgen Receptor Gene Silencing. Cell Rep, 17, 125-136.

46721 Herbst, E.A.F. and Holloway, G.P. (2015) Exercise Training Normalizes Mitochondrial

468 Respiratory Capacity within the Striatum of the R6/1 Model of Huntington's Disease.

469 Neuroscience, 303, 515-523.

47022 Ji, E.S., Kim, Y.M., Shin, M.S., Kim, C.J., Lee, K.S., Kim, K., Ha, J. and Chung, Y.R.

471 (2015) Treadmill exercise enhances spatial learning ability through suppressing hippocampal 472 apoptosis in Huntington's disease rats. J Exerc Rehabil, 11, 133-139.

47323 Corrochano, S., Blanco, G., Williams, D., Wettstein, J., Simon, M., Kumar, S., Moir, L., 474 Agnew, T., Stewart, M., Landman, A. et al. (2018) A genetic modifier suggests that endurance 475 exercise exacerbates Huntington's disease. Hum Mol Genet, 27, 1723-1731.

47624 Bonini, N.M. and Fortini, M.E. (2003) Human neurodegenerative disease modeling using 477 Drosophila. Annu Rev Neurosci, 26, 627-656.

47825 Perrimon, N., Bonini, N.M. and Dhillon, P. (2016) Fruit flies on the front line: the 479 translational impact of Drosophila. Dis Model Mech, 9, 229-231.

48026 Pandey, M. and Rajamma, U. (2018) Huntington's disease: the coming of age. J Genet, $48197,649-664$.

48227 Ueyama, M. and Nagai, Y. (2018) Repeat Expansion Disease Models. Adv Exp Med 483 Biol, 1076, 63-78.

48428 Sujkowski, A. and Wessells, R. (2018) Using Drosophila to Understand Biochemical and 485 Behavioral Responses to Exercise. Exerc Sport Sci Rev, 46, 112-120. 
487 in young Drosophila melanogaster reduces age-related decline in mobility and cardiac

488 performance. PLoS One, 4, e5886.

48930 Sujkowski, A., Saunders, S., Tinkerhess, M., Piazza, N., Jennens, J., Healy, L., Zheng,

490 L. and Wessells, R. (2012) dFatp regulates nutrient distribution and long-term physiology in

491 Drosophila. Aging Cell, 11, 921-932.

49231 Laker, R.C., Xu, P., Ryall, K.A., Sujkowski, A., Kenwood, B.M., Chain, K.H., Zhang, M.,

493 Royal, M.A., Hoehn, K.L., Driscoll, M. et al. (2014) A novel MitoTimer reporter gene for

494 mitochondrial content, structure, stress, and damage in vivo. J Biol Chem, 289, 12005-12015.

49532 Sujkowski, A., Bazzell, B., Carpenter, K., Arking, R. and Wessells, R.J. (2015)

496 Endurance exercise and selective breeding for longevity extend Drosophila healthspan by

497 overlapping mechanisms. Aging (Albany NY), 7, 535-552.

49833 Damschroder, D., Reynolds, C. and Wessells, R. (2018) Drosophila tafazzin mutants

499 have impaired exercise capacity. Physiol Rep, 6.

50034 Damschroder, D., Cobb, T., Sujkowski, A. and Wessells, R. (2018) Drosophila

501 Endurance Training and Assessment of Its Effects on Systemic Adaptations. Bio-protocol, 8, 502 e3037.

50335 Sujkowski, A., Spierer, A.N., Rajagopalan, T., Bazzell, B., Safdar, M., Imsirovic, D., 504 Arking, R., Rand, D.M. and Wessells, R. (2018) Mito-nuclear interactions modify Drosophila 505 exercise performance. Mitochondrion, in press.

50636 Sujkowski, A., Spierer, A.N., Rajagopalan, T., Bazzell, B., Safdar, M., Imsirovic, D., 507 Arking, R., Rand, D.M. and Wessells, R. (2019) Mito-nuclear interactions modify Drosophila 508 exercise performance. Mitochondrion, 47, 188-205.

50937 Wessells, R., Safdar, M. and Sujkowski, A. (2018) Ram, J. (ed.), In Conn's Handbook of 510 Models for Human Aging, in press. 
51138 Tinkerhess, M.J., Healy, L., Morgan, M., Sujkowski, A., Matthys, E., Zheng, L. and

512 Wessells, R.J. (2012) The Drosophila PGC-1alpha homolog spargel modulates the

513 physiological effects of endurance exercise. PLoS One, 7, e31633.

51439 Kim, M., Sujkowski, A., Namkoong, S., Gu, B., Cobb, T., Kim, B., Kowalsky, A.H., Cho,

515 C.S., Semple, I., Ro, S.H. et al. (2020) Sestrins are evolutionarily conserved mediators of

516 exercise benefits. Nat Commun, 11, 190.

51740 Tsou, W.L., Burr, A.A., Ouyang, M., Blount, J.R., Scaglione, K.M. and Todi, S.V. (2013)

518 Ubiquitination regulates the neuroprotective function of the deubiquitinase ataxin-3 in vivo. J Biol

519 Chem, 288, 34460-34469.

52041 Tsou, W.L., Hosking, R.R., Burr, A.A., Sutton, J.R., Ouyang, M., Du, X., Gomez, C.M.

521 and Todi, S.V. (2015) DnaJ-1 and karyopherin alpha-3 suppress degeneration in a new

522 Drosophila model of Spinocerebellar Ataxia Type 6. Hum Mol Genet, in press.

52342 Tsou, W.L., Ouyang, M., Hosking, R.R., Sutton, J.R., Blount, J.R., Burr, A.A. and Todi,

524 S.V. (2015) The deubiquitinase ataxin-3 requires Rad23 and DnaJ-1 for its neuroprotective role

525 in Drosophila melanogaster. Neurobiol Dis, 82, 12-21.

52643 Costa, M.D., Ashraf, N.S., Fischer, S., Yang, Y., Schapka, E., Joshi, G., McQuade, T.J.,

527 Dharia, R.M., Dulchavsky, M., Ouyang, M. et al. (2016) Unbiased screen identifies aripiprazole

528 as a modulator of abundance of the polyglutamine disease protein, ataxin-3. Brain, in press.

52944 Tsou, W.L., Qiblawi, S.H., Hosking, R.R., Gomez, C.M. and Todi, S.V. (2016)

530 Polyglutamine length-dependent toxicity from alpha1ACT in Drosophila models of

531 spinocerebellar ataxia type 6. Biol Open, 5, 1770-1775.

53245 Sutton, J.R., Blount, J.R., Libohova, K., Tsou, W.L., Joshi, G.S., Paulson, H.L., do

533 Carmo Costa, M., Scaglione, M.K. and Todi, S.V. (2017) Interaction of the polyglutamine protein

534 ataxin-3 with Rad23 regulates toxicity in Drosophila models of Spinocerebellar Ataxia Type 3.

535 Hum Mol Genet, in press. 
53646 Ristic, G., Sutton, J.R., Libohova, K. and Todi, S.V. (2018) Toxicity and aggregation of

537 the polyglutamine disease protein, ataxin-3 is regulated by its binding to VCP/p97 in Drosophila

538 melanogaster. Neurobiol Dis, 116, 78-92.

53947 Johnson, S.L., Blount, J.R., Libohova, K., Ranxhi, B., Paulson, H.L., Tsou, W.L. and

540 Todi, S.V. (2019) Differential toxicity of ataxin-3 isoforms in Drosophila models of

541 Spinocerebellar Ataxia Type 3. Neurobiol Dis, 132, 104535.

54248 Jackson, G.R., Salecker, I., Dong, X., Yao, X., Arnheim, N., Faber, P.W., MacDonald,

543 M.E. and Zipursky, S.L. (1998) Polyglutamine-expanded human huntingtin transgenes induce

544 degeneration of Drosophila photoreceptor neurons. Neuron, 21, 633-642.

54549 Fernandez-Funez, P., Nino-Rosales, M.L., de Gouyon, B., She, W.C., Luchak, J.M.,

546 Martinez, P., Turiegano, E., Benito, J., Capovilla, M., Skinner, P.J. et al. (2000) Identification of

547 genes that modify ataxin-1-induced neurodegeneration. Nature, 408, 101-106.

54850 Romero, E., Cha, G.H., Verstreken, P., Ly, C.V., Hughes, R.E., Bellen, H.J. and Botas,

549 J. (2008) Suppression of neurodegeneration and increased neurotransmission caused by

550 expanded full-length huntingtin accumulating in the cytoplasm. Neuron, 57, 27-40.

$55151 \quad$ Hsu, T.C., Wang, C.K., Yang, C.Y., Lee, L.C., Hsieh-Li, H.M., Ro, L.S., Chen, C.M., Lee-

552 Chen, G.J. and Su, M.T. (2014) Deactivation of TBP contributes to SCA17 pathogenesis. Hum

553 Mol Genet, 23, 6878-6893.

55452 Rosas-Arellano, A., Estrada-Mondragon, A., Pina, R., Mantellero, C.A. and Castro, M.A.

555 (2018) The Tiny Drosophila Melanogaster for the Biggest Answers in Huntington's Disease. Int J

556 Mol Sci, 19.

55753 Brand, A.H., Manoukian, A.S. and Perrimon, N. (1994) Ectopic expression in Drosophila.

558 Methods in cell biology, 44, 635-654.

55954 Johnson, S.L., Ranxhi, B., Libohova, K., Tsou, W.L. and Todi, S.V. (2020) Ubiquitin-

560 interacting motifs of ataxin-3 regulate its polyglutamine toxicity through Hsc70-4-dependent

561 aggregation. Elife, 9. 
563 protocol and longitudinal performance assays for Drosophila melanogaster. $J$ Vis Exp, in press.

$56456 \quad$ Chen, S.D., Yang, J.L., Lin, T.K. and Yang, D.I. (2019) Emerging Roles of Sestrins in

565 Neurodegenerative Diseases: Counteracting Oxidative Stress and Beyond. J Clin Med, 8.

56657 Mahalakshmi, B., Maurya, N., Lee, S.D. and Bharath Kumar, V. (2020) Possible

567 Neuroprotective Mechanisms of Physical Exercise in Neurodegeneration. Int J Mol Sci, 21.

56858 Lee, J.H., Budanov, A.V., Park, E.J., Birse, R., Kim, T.E., Perkins, G.A., Ocorr, K.,

569 Ellisman, M.H., Bodmer, R., Bier, E. et al. (2010) Sestrin as a feedback inhibitor of TOR that

570 prevents age-related pathologies. Science, 327, 1223-1228.

57159 Wardman, J.H., Henriksen, E.E., Marthaler, A.G., Nielsen, J.E. and Nielsen, T.T. (2020)

572 Enhancement of Autophagy and Solubilization of Ataxin-2 Alleviate Apoptosis in Spinocerebellar

573 Ataxia Type 2 Patient Cells. Cerebellum, 19, 165-181.

57460 Johnson, S.L., Blount, J.R., Libohova, K., Ranxhi, B., Paulson, H.L., Tsou, W.L. and

575 Todi, S.V. (2019) Differential toxicity of ataxin-3 isoforms in Drosophila models of

576 Spinocerebellar Ataxia Type 3. Neurobiology of Disease, 132.

57761 Scoles, D.R. and Pulst, S.M. (2018) Spinocerebellar Ataxia Type 2. Adv Exp Med Biol,

578 1049, 175-195.

57962 Kim, H., An, S., Ro, S.H., Teixeira, F., Park, G.J., Kim, C., Cho, C.S., Kim, J.S., Jakob,

580 U., Lee, J.H. et al. (2015) Janus-faced Sestrin2 controls ROS and mTOR signalling through two 581 separate functional domains. Nature Communications, 6.

58263 Sujkowski, A., Gretzinger, A., Soave, N., Todi, S.V. and Wessells, R. (2020) Alpha- and 583 beta-adrenergic octopamine receptors in muscle and heart are required for Drosophila exercise 584 adaptations. PLoS Genet, 16, e1008778.

58564 Budanov, A.V. and Karin, M. (2008) p53 target genes sestrin1 and sestrin2 connect 586 genotoxic stress and mTOR signaling. Cell, 134, 451-460. 
65 Singh, P. and Chowdhuri, D.K. (2018) Modulation of sestrin confers protection to $\mathrm{Cr}(\mathrm{VI})$

588 induced neuronal cell death in Drosophila melanogaster. Chemosphere, 191, 302-314.

58966 Reddy, K., Cusack, C.L., Nnah, I.C., Khayati, K., Saqcena, C., Huynh, T.B., Noggle,

590 S.A., Ballabio, A. and Dobrowolski, R. (2016) Dysregulation of Nutrient Sensing and

591 CLEARance in Presenilin Deficiency. Cell Rep, 14, 2166-2179.

59267 Fatokun, A.A., Stone, T.W. and Smith, R.A. (2008) Oxidative stress in

593 neurodegeneration and available means of protection. Front Biosci, 13, 3288-3311.

59468 Mangiarini, L., Sathasivam, K., Seller, M., Cozens, B., Harper, A., Hetherington, C.,

595 Lawton, M., Trottier, Y., Lehrach, H., Davies, S.W. et al. (1996) Exon 1 of the HD gene with an

596 expanded CAG repeat is sufficient to cause a progressive neurological phenotype in transgenic

597 mice. Cell, 87, 493-506.

59869 van Dellen, A., Cordery, P.M., Spires, T.L., Blakemore, C. and Hannan, A.J. (2008)

599 Wheel running from a juvenile age delays onset of specific motor deficits but does not alter

600 protein aggregate density in a mouse model of Huntington's disease. Bmc Neuroscience, 9.

60170 Paulson, H.L., Das, S.S., Crino, P.B., Perez, M.K., Patel, S.C., Gotsdiner, D., Fischbeck,

602 K.H. and Pittman, R.N. (1997) Machado-Joseph disease gene product is a cytoplasmic protein

603 widely expressed in brain. Ann Neurol, 41, 453-462.

60471 Blount, J.R., Libohova, K., Marsh, G.B., Sutton, J.R. and Todi, S.V. (2018) Expression

605 and Regulation of Deubiquitinase-Resistant, Unanchored Ubiquitin Chains in Drosophila. Sci

$606 \operatorname{Rep}, \mathbf{8}, 8513$.

60772 Sutton, J.R., Blount, J.R., Libohova, K., Tsou, W.L., Joshi, G.S., Paulson, H.L., Costa, 608 M.D.C., Scaglione, K.M. and Todi, S.V. (2017) Interaction of the polyglutamine protein ataxin-3 609 with Rad23 regulates toxicity in Drosophila models of Spinocerebellar Ataxia Type 3. Hum Mol 610 Genet, 26, 1419-1431. 
bioRxiv preprint doi: https://doi.org/10.1101/2021.12 06.471345; this version posted December 7, 2021. The copyright holder for this preprint

(which was not certified by peer review) is the author/funder, who has granted bioRxiv a license to display the preprint in perpetuity. It is made available under aCC-BY 4.0 International license.

61173 Damschroder, D., Cobb, T., Sujkowski, A. and Wessells, R. (2017) Drosophila

612 Endurance Training and Assessment and Its Effects on Systemic Adaptations. BioProtocols, in

613 press.

614

615

616 
Figure 1: Endurance exercise differentially affects mobility in Drosophila models of

620 Spinocerebellar Ataxia. (A) Table of Spinocerebellar Ataxia (SCA) models used in this study.

621 (B) Timeline of endurance exercise program and assessment of physiology and disease protein

622 levels. (C-E) Representative climbing speed images in Drosophila models of (C) SCA2, (D)

623 SCA3 and (E) SCA6. Photos taken 2 seconds after inducing negative geotaxis response in 4-

624 week-old flies, after endurance exercise is complete.

Figure 2: Endurance exercise differentially affects mobility and disease protein levels

Drosophila models of SCA. (A) Flies ectopically expressing polyQ-expanded ATXN2 in adult neurons (RU+) have lower climbing speed than unexercised, uninduced control flies (RU- UN) by adult week 4 . Exercise fully rescues climbing speed to the level of exercised, uninduced control flies (compare RU- EX to RU+ EX). (B) Flies expressing polyQ-expanded ATXN2 in adult neurons $(\mathrm{RU}+)$ have similar endurance to uninduced control flies (RU-) whether exercised or not. (C) Exercise reduces ATXN2 levels in flies ectopically expressing polyQ-expanded ATXN2 in adult neurons. (D-E) Flies ectopically expressing polyQ-expanded ATXN3 in adult

634 neurons (RU+) have similar (D) climbing speed and $(E)$ endurance to uninduced, unexercised

635 control flies and fail to improve either (D) climbing speed or (E) endurance with exercise. (F)

636 Exercise does not affect ATXN3 levels in flies expressing polyQ-expanded ATXN3 in adult

637 neurons. (G) Flies ectopically expressing polyQ-expanded $\alpha 1 A C T$ in adult neurons (RU+) have

638 lower climbing speed than unexercised, uninduced control flies (RU- UN) by adult week 3, and

639 exercise partially rescues climbing speed, although not to the level of exercised, uninduced

640 control flies. (H) Exercise improves endurance in flies expressing polyQ-expanded $\alpha 1 A C T$ in

641 adult neurons (RU+ EX), but endurance is not as good as exercised, uninduced control siblings 
642 (compare RU- EX to RU+ EX). Mobility and endurance experiments performed in triplicate.

$643 \mathrm{n} \geq 100$ (climbing speed), $\mathrm{n} \geq 8$ vials of 20 flies (endurance). Error bars indicate $+/-S D$.

645 Figure 3: Expression of Atxn2 in adult neurons does not negatively impact lifespan.

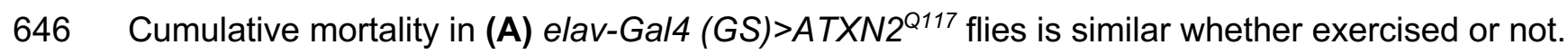

647 (B) elav-Gal4 (GS)>ATXN3 ${ }^{Q 77}$ flies have reduced lifespan compared to uninduced controls, and 648 exercise does not negatively affect either group. (C) elav-Gal4 (GS)>a1ACT $T^{Q 70}$ flies have lower 649 lifespan than uninduced controls, and exercise reduces lifespan further. $\mathrm{n} \geq 231$.

Figure 4: dSesn expression improves early death and low mobility in SCA2 flies,

652 concurrent with reductions in disease protein. Female (A, B) and male (D, E) flies

653 ubiquitously expressing polyQ-expanded ATXN2 (red lines) have early death (A, D) and lower

654 climbing speed (B, E) than age-matched background control flies (blue and black lines). dSesn

655 expression in flies also expressing polyQ-expanded ATXN2 (green lines) partially rescues early

656 death (A, D) and fully rescues decreased mobility (B, E). ATXN2 levels are lower in both female

657 (C) and male (F) flies ubiquitously expressing both dSesn and polyQ-expanded ATXN2.

658 Survival and mobility experiments performed in triplicate. $n \geq 200$ (survival). $n \geq 100$ (climbing

659 speed). Error bars indicate +/-SD. Representative Western blots from four biological repetitions, 660 quantified in Figure 6.

Figure 5: Oxidoreductase function is dispensable for mobility-extending effects of dSesn

663 in flies expressing polyQ-expanded ATXN2. Ubiquitous expression of dSesn harboring a

664 point mutation that abolishes oxidoreductase activity ( $d S e s n^{C S}$, orange lines) exacerbates early

665 death in SCA2 model flies (compare orange lines to red lines) in both females (A) and males

666 (D). In contrast, climbing speed is partially rescued in the same female flies (expressing both

$667 \mathrm{dSesn}{ }^{\mathrm{CS}}$ and polyQ-expanded ATXN2, in orange) (B) and fully rescued in males (E). (C, F) Wild- 
type dSesn expression reduces ATXN2 levels, but dSesn ${ }^{\mathrm{CS}}$ expression does not. Survival and mobility experiments performed in triplicate. $n \geq 200$ (survival). $n \geq 100$ (climbing speed). Survival and climbing speed graphs depicted from same repetition depicted in Figure 2. Error bars

671 indicate +/-SD. Representative Western blots from four biological repetitions, quantified in

\section{Figure 6.}

Figure 6: Interaction with mTOR is required for dSesn to improve survival and mobility in

675 flies expressing polyQ-expanded ATXN2. Ubiquitous expression of dSesn harboring two 676 separate mutations that abolish mTORC interaction $d \operatorname{Sesn}^{D A}(\mathbf{A}-\mathrm{D}), d \operatorname{Sesn}^{D D A A}(\mathbf{E}-\mathrm{H})$ orange

677 lines) exacerbates early death (A,E, females, C,G, males) and fails to rescue mobility (B,F, 678 females, D,H, males) in SCA2 model flies. Survival and mobility experiments performed in triplicate. Survival and climbing speed graphs depicted from same repetition depicted in Figure 2. $n \geq 200$ (survival). $n \geq 100$ (climbing speed). Error bars indicate $+/-S D$.

Figure 7: Wild-type dSesn expression reduces disease protein and increases autophagy in flies expressing polyQ-expanded ATXN2. Ubiquitous expression of dSesn significantly reduces ATXN2 levels (A-C, n=4) and increases Atg1a;Atg2a ratios (C-E, n=3). (F) Genotypes in representative Western blot and from independent biological replicates, quantified in A, B, D, and E. Select individual Western blots repetitions shown in previous Figures 4 and 5. Bracket 687 indicates quantifications from $A, B, D$, and $E$.

690 ATXN2. Exercised female flies expressing two copies of polyQ expanded SCA2 ubiquitously (A) 691 or in adult neurons (C) have increased survival compared to age-matched, unexercised siblings, 692 while exercised male flies expressing two copies of polyQ expanded SCA2 ubiquitously (B) or in 693 adult neurons (D) trend toward increased survival only in the first 25 days, the period in which 
694 flies are still undergoing exercise training. Brackets indicate exercise training period. P-values

695 indicate log-rank for entire survival curve, $n \geq 170$, performed in duplicate.

Figure 9: Proposed model of the effects of exercise and dSesn overexpression on SCA2

flies. Sestrin's known functions and activation by exercise have been established previously

$699(39,62)$. Wild type Sesn overexpression or exercise can activate autophagy and reduce disease

700 protein levels, improving function.

701

Supplementary Figure legends

703

Supplementary Video SV1: SCA2 model flies increase motility with exercise. Video of 4week-old uninduced control and SCA2 model flies taken following 3 weeks of ramped endurance exercise. Order of vials depicted, left to right: Vial 1-uninduced, exercised control, Vials 2,3-SCA2 model, exercised, Vials 4,5-SCA2 model, unexercised.

708 polyQ-80 repeat in adult neurons have lower climbing speed than age-matched, uninduced control flies and do not increase speed with exercise. Mobility and endurance experiments

714 performed in triplicate. $n \geq 100$ (climbing speed), $n \geq 8$ vials of 20 flies (endurance). Error bars 715 indicate +/-SD.

718 Supplementary Figure S2: Ubiquitous dSesn expression in wild-type flies does not 719 account for survival differences observed in flies expressing polyQ-expanded ATXN2. 
720 Survival curves in female (A) and male (B) flies expressing either wild-type dSesn (green),

$721 \mathrm{dSesn}^{\mathrm{CS}}$ (red), dSesn ${ }^{\mathrm{DA}}$ (blue), dSesn ${ }^{\mathrm{DDAA}}$ (purple) or background control (black). Flies

722 ubiquitously expressing dSesn mutations have similar or lower survival than flies ubiquitously

723 expressing wild type dSesn. $n \geq 97$.

\section{Source data-all figures}

726 Tab delimited excel file containing raw data for all figures and supplementary figures.

727 Uncropped, unedited Western blot data are labeled and included as separate jpegs. 
Climbing Speed

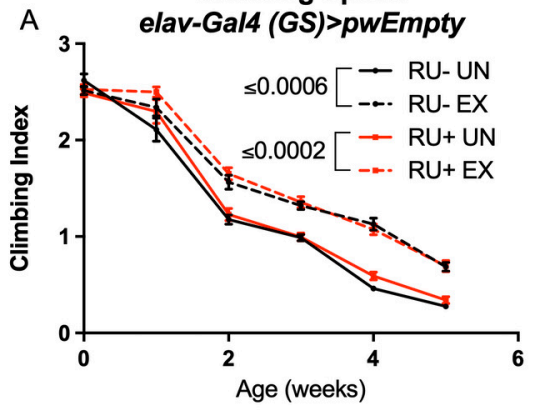

Day 25 Runspan

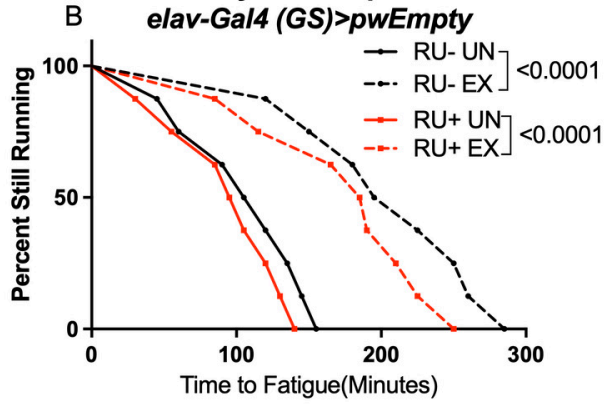

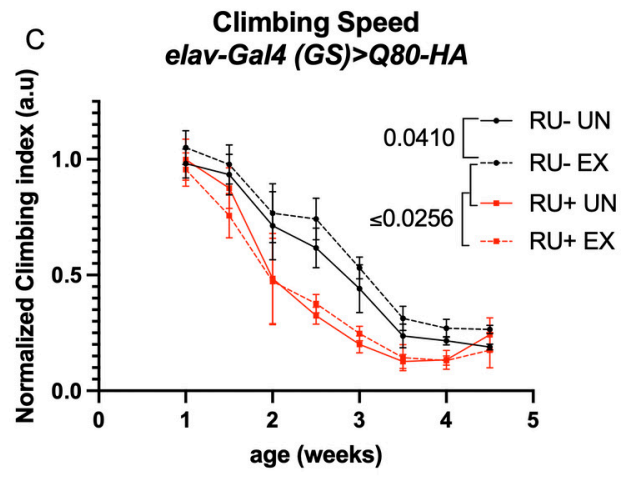


A

Deaths

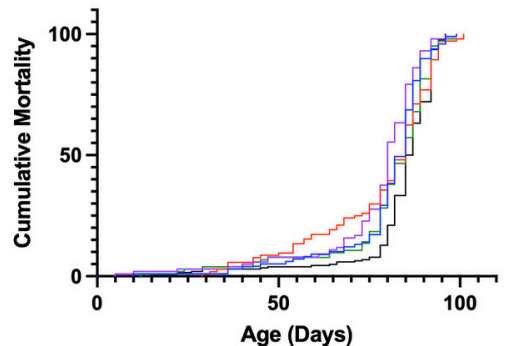

- $w^{1118 ; s q h>d S e s n}{ }^{c S}$ 우

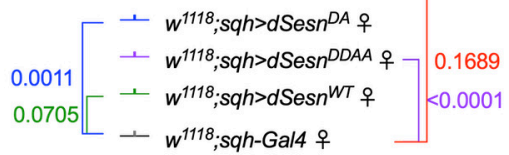

B

Deaths

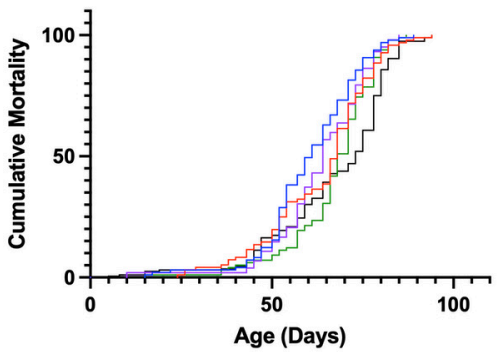

- $w^{1118}$;sqh $>d \operatorname{Sesn}^{C S} \sigma^{1}$

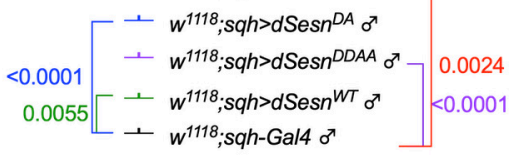

